\title{
OPEN Author Correction: Optimization of Molecules via Deep Reinforcement Learning
}

\section{Zhenpeng Zhou $\mathbb{D}^{\mathrm{D}}$, Steven Kearnes $\mathbb{B}$, Li Li, Richard N. Zare \& Patrick Riley $\mathbb{1}$}

Correction to: Scientific Reports https://doi.org/10.1038/s41598-019-47148-x, published online 24 July 2019

This Article contains errors.

Penalized $\log \mathrm{P}$ is reported in this Article for our methods in a non-normalised form. Previous research (which we compared to in the original Tables 1 and 2), starting with JT-VAE ${ }^{1}$ normalized the $\log P$ to be zero mean and unit standard deviation based on the training set. To correctly compare with previous research, our penalized logP was recalculated into a normalised form. This resulted in changes of numerical values for Penalized $\log \mathrm{P}$ in Table 1, and numerical values for MolDQN-naïve and MolDQN-bootstrap Improvement in Table 2. The corrected versions of these tables are included below as Tables 1 and 2.

\begin{tabular}{|c|c|c|c|c|c|c|c|c|}
\hline & \multicolumn{4}{|c|}{ Penalized $\log P$} & \multicolumn{4}{|l|}{ QED } \\
\hline & 1 st & 2nd & 3 rd & Validity & 1 st & 2nd & $3 r d$ & Validity \\
\hline random walk ${ }^{\mathrm{a}}$ & -0.65 & -1.72 & -1.88 & $100 \%$ & 0.64 & 0.56 & 0.56 & $100 \%$ \\
\hline greedy $^{b}$ & 9.05 & - & - & $100 \%$ & 0.39 & - & - & $100 \%$ \\
\hline$\varepsilon$-greedy, $\varepsilon=0.1^{\mathrm{b}}$ & 9.10 & 9.05 & 9.05 & $100 \%$ & 0.914 & 0.910 & 0.906 & $100 \%$ \\
\hline $\mathrm{JT}^{\mathrm{V}} \mathrm{VAE}^{\mathrm{c}}$ & 5.30 & 4.93 & 4.49 & $100 \%$ & 0.925 & 0.911 & 0.910 & $100 \%$ \\
\hline ORGAN $^{c}$ & 3.63 & 3.49 & 3.44 & $0.4 \%$ & 0.896 & 0.824 & 0.820 & $2.2 \%$ \\
\hline $\mathrm{GCPN}^{\mathrm{c}}$ & 7.98 & 7.85 & 7.80 & $100 \%$ & 0.948 & 0.947 & 0.946 & $100 \%$ \\
\hline MolDQN-naive & 8.69 & 8.68 & 8.67 & $100 \%$ & 0.934 & 0.931 & 0.930 & $100 \%$ \\
\hline MolDQN-bootstrap & 9.01 & 9.01 & 8.99 & $100 \%$ & 0.948 & 0.944 & 0.943 & $100 \%$ \\
\hline MolDQN-bootstrap & - & - & - & - & 0.948 & 0.948 & 0.948 & $100 \%$ \\
\hline
\end{tabular}

Table 1. Top three unique molecule property scores found by each method. " "random walk" is a baseline that chooses a random action for each step. " "greedy" is a baseline that chooses the action that leads to the molecule with the highest reward for each step. " $\varepsilon$-greedy" follows the "random" policy with probability $\varepsilon$, and "greedy" policy with probability $1-\varepsilon$. In contrast, the $\varepsilon$-greedy MolDQN models choose actions based on predicted $\mathrm{Q}$-values rather than rewards. ${ }^{\mathrm{c}}$ values are reported in You et al. ${ }^{2}$.

\begin{tabular}{|l|l|l|l|l|l|l|l|l|}
\hline \multirow{2}{*}{$\delta$} & \multicolumn{3}{|l|}{ JT-VAE $^{\mathbf{a}}$} & \multicolumn{2}{l|}{ GCPN $^{\mathbf{a}}$} & \multicolumn{2}{l|}{ MolDQN-naive } & \multicolumn{2}{l|}{ MolDQN-bootstrap } \\
\cline { 2 - 11 } & Improvement & Success & Improvement & Success & Improvement & Success & Improvement & Success \\
\hline 0.0 & $1.91 \pm 2.04$ & $97.5 \%$ & $4.20 \pm 1.28$ & $100 \%$ & $4.83 \pm 1.30$ & $100 \%$ & $4.88 \pm 1.30$ & $100 \%$ \\
\hline 0.2 & $1.68 \pm 1.85$ & $97.1 \%$ & $4.12 \pm 1.19$ & $100 \%$ & $3.79 \pm 1.32$ & $100 \%$ & $3.80 \pm 1.30$ & $100 \%$ \\
\hline 0.4 & $0.84 \pm 1.45$ & $83.6 \%$ & $2.49 \pm 1.30$ & $100 \%$ & $2.34 \pm 1.18$ & $100 \%$ & $2.44 \pm 1.25$ & $100 \%$ \\
\hline 0.6 & $0.21 \pm 0.71$ & $46.4 \%$ & $0.79 \pm 0.63$ & $100 \%$ & $1.40 \pm 0.92$ & $100 \%$ & $1.30 \pm 0.98$ & $100 \%$ \\
\hline
\end{tabular}

Table 2. Mean and standard deviation of penalized $\log P$ improvement in constrained optimization tasks. $\delta$ is the threshold of the similarity constraint $\operatorname{SIM}\left(\mathrm{m}, \mathrm{m}_{0}\right) \geq \delta$. The success rate is the percentage of molecules satisfying the similarity constraint. ${ }^{\mathrm{a}}$ values are reported in You et al. ${ }^{2}$. 
Additionally, in the Discussion, subsection "Constrained optimization”:

"Using Welch's $t$-test ${ }^{30}$ for $N=800$ molecules, we found that both variants of MolDQN gives a highly statistically significant improvement over GCPN for all values of $\delta$ with $t<-8$. The bootstrap variant also significantly outperforms the naive model (except for $\delta=0.2$ ) with $t<-3$."

should read:

"Using Welch's $t$-test ${ }^{30}$, we found that on $\delta=0.2$, both variants of MolDQN gives a statistically significant lower improvement comparing to GCPN with $P<1 \mathrm{e}-7$; on $\delta=0.4$, the differences are insignificant at a $1 \%$ level with $P=0.016$ for MolDQN naive and $P=0.43$ for MolDQN bootstrap; on $\delta=0.0$ and 0.6 , both variants of MolDQN gives a statistically significant higher improvement comparing to GCPN with $P<1 \mathrm{e}-22$. The differences between two variants of MolDQN are statistically insignificant at a $1 \%$ level with $P>0.036$."

Furthermore, Figure S7 was updated. The corrected version is shown below as Figure 1.

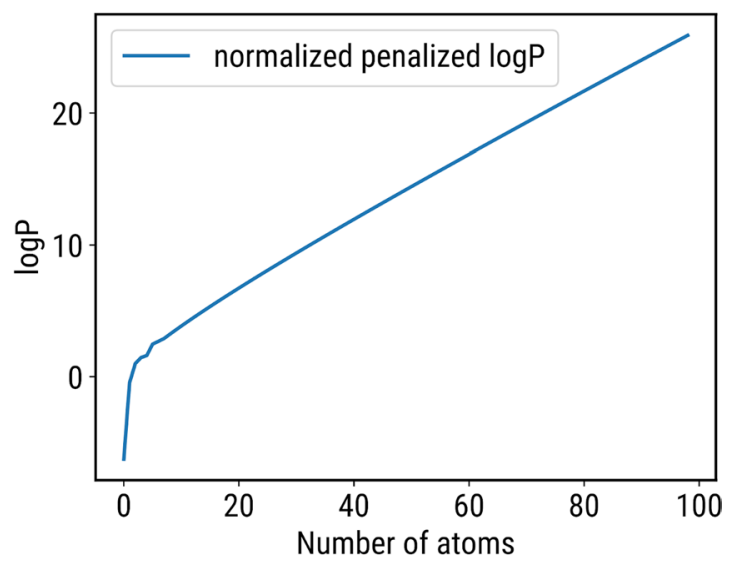

Figure 1 .

Lastly, References 13 and 18 were incorrectly given as:

13. Jin, W., Barzilay, R. \& Jaakkola, T. Junction tree variational autoencoder for molecular graph generation. arXiv preprint arXiv:1802.04364 (2018).

18. You, J., Liu, B., Ying, R., Pande, V. \& Leskovec, J. Graph convolutional policy network for goal-directed molecular graph generation. arXiv preprint arXiv:1806.02473 (2018).

The correct references are listed below as references 1 and 2 .

These changes do not affect the conclusions of the Article.

\section{References}

1. Jin, W., Barzilay, R. \& Jaakkola, T. Junction Tree Variational Autoencoder for Molecular Graph Generation. In Proceedings of the 35th International Conference on Machine Learning (eds. Dy, J. \& Krause, A.) vol. 80, 2323-2332 (PMLR, 2018).

2. You, J., Liu, B., Ying, Z., Pande, V. \& Leskovec, J. Graph Convolutional Policy Network for Goal-Directed Molecular Graph Generation. In Advances in Neural Information Processing Systems 31 (eds. Bengio, S. et al.) 6410-6421 (Curran Associates, Inc., 2018).

(c) (i) Open Access This article is licensed under a Creative Commons Attribution 4.0 International License, which permits use, sharing, adaptation, distribution and reproduction in any medium or format, as long as you give appropriate credit to the original author(s) and the source, provide a link to the Creative Commons license, and indicate if changes were made. The images or other third party material in this article are included in the article's Creative Commons license, unless indicated otherwise in a credit line to the material. If material is not included in the article's Creative Commons license and your intended use is not permitted by statutory regulation or exceeds the permitted use, you will need to obtain permission directly from the copyright holder. To view a copy of this license, visit http://creativecommons.org/licenses/by/4.0/.

(C) The Author(s) 2020 